\title{
Unsupervised SAR Image Segmentation Based on a Hierarchical TMF Model in the Discrete Wavelet Domain for Sea Area Detection
}

\author{
Jiajing Wang, ${ }^{1,2}$ Shuhong Jiao, ${ }^{1}$ Lianyang Shen, ${ }^{3}$ Zhenyu Sun, ${ }^{4}$ and Lin Tang ${ }^{1}$ \\ ${ }^{1}$ College of Information and Communication Engineering, Harbin Engineering University, Harbin 150001, China \\ ${ }^{2}$ No. 92677 Unit of PLA, Dalian 116001, China \\ ${ }^{3}$ Naval Armaments Department Military Representative Office, Shenyang 110000, China \\ ${ }^{4}$ No. 91550 Unit of PLA, Dalian 116001, China \\ Correspondence should be addressed to Jiajing Wang; 601770950@qq.com
}

Received 9 August 2014; Accepted 8 October 2014; Published 4 November 2014

Academic Editor: Xiaojie Su

Copyright (C) 2014 Jiajing Wang et al. This is an open access article distributed under the Creative Commons Attribution License, which permits unrestricted use, distribution, and reproduction in any medium, provided the original work is properly cited.

\begin{abstract}
Unsupervised synthetic aperture radar (SAR) image segmentation is a fundamental preliminary processing step required for sea area detection in military applications. The purpose of this step is to classify large image areas into different segments to assist with identification of the sea area and the ship target within the image. The recently proposed triplet Markov field (TMF) model has been successfully used for segmentation of nonstationary SAR images. This letter presents a hierarchical TMF model in the discrete wavelet domain of unsupervised SAR image segmentation for sea area detection, which we have named the wavelet hierarchical TMF (WHTMF) model. The WHTMF model can precisely capture the global and local image characteristics in the two-pass computation of posterior distribution. The multiscale likelihood and the multiscale energy function are constructed to capture the intrascale and intrascale dependencies in a random field $(X, U)$. To model the SAR data related to radar backscattering sources, the Gaussian distribution is utilized. The effectiveness of the proposed model for SAR image segmentation is evaluated using synthesized and real SAR data.
\end{abstract}

\section{Introduction}

SAR is an active remote sensing system that generates and transmits microwave electromagnetic radiation to the surface of a target region [1]. It has been widely applied in national defense applications since SAR imaging is not influenced by weather conditions, geographical location, or time.

For a special application of sea area detection, SAR image is provided to detect a ship target in the sea area. And in this type of application, the SAR image needs to be firstly segmented to identify the sea area within the image. Therefore, SAR image segmentation is an important problem that requires further investigation.

Based on a review of recent studies, segmentation methods can be divided into multiple categories including featurebased methods [2], structure-based methods [3], and modelbased methods. Among these methods, the Markov random field (MRF) $[4,5]$ model is very popular in the research community, since it is acknowledged as a powerful tool for the segmentation of SAR images. The MRF model can suppress speckle noise during the process of image segmentation and produce better results than other methods. Several studies have applied the MRF model to various problems, including the detection of oil spill within SAR images [6] and modified MRF-based SAR image segmentation [7, 8]. However, real SAR images are often nonstationary, especially in object detection applications, and the MRF model does not take the nonstationary nature of SAR images into consideration. This limitation makes it unsuitable for nonstationary SAR images.

Benboudjema and Pieczynski were the first to propose the triplet Markov field (TMF) model and introduced an auxiliary random field to deal with nonstationary image segmentation [9]. The TMF model has achieved promising results tackling SAR image segmentation. For example, Wang et al. 
proposed a change-detection method for segmentation based on the TMF model. She redefined the third field $U$ in the TMF model to describe the nonstationary textural similarity between two multitemporal SAR images [10]. In another study, Wang et al. used a higher order neighborhood system in the TMF model to improve the modeling ability for complex priors [11]. Lian et al. proposed an unsupervised SAR image segmentation algorithm based on a conditional TMF framework which combines the advantages of both CRF and TMF [12]. However, these TMF-based, segmentation algorithms are employed at a pixel level in the spatial domain, which means iterative calculations are required to obtain an approximate result. Taking the large calculation requirement into account, Gan et al. used the TMF model with edge location for fast unsupervised segmentation of SAR images [13]. The computational efficiency is significantly enhanced but this method is also in the spatial domain; therefore the impact of reducing the computation time is a loss of local information.

In this letter, a novel unsupervised SAR image segmentation method is proposed that is based on a hierarchical TMF in the discrete wavelet domain. The multiresolution characteristic of the discrete wavelet transform is used to represent the observed field of the SAR image as a series of discrete wavelet coefficients, so that each labeled field of the corresponding resolution can use the characteristic of the corresponding scale. A hierarchical algorithm in the discrete wavelet domain defines the relationship between different scales using a model that incorporates the global and local characteristics of an image at different scales, which can improve the efficiency of image segmentation. The WHTMF method in this paper combines the advantages of both the hierarchical algorithm and the TMF model to deal with the problem of insufficient local statistical information. Unsupervised segmentation is popular in recent years since training data is not required for parameter estimation, which is automatically accomplished and is conducive to automation of the sea area detection system. The experimental results suggest that the proposed method can improve the accuracy of SAR image segmentation.

\section{Triplet Markov Field}

The TMF model is developed based on the classical hidden Markov fields (HMF) mode and the pairwise Markov field (PMF) model [14]. The TMF introduces a third random field $U=\left(U_{s}\right)_{s \in S}$ and assumes the TMF $T=(X, U, Y)$. In contrast to the HMF and PMF, the third field can have some physical interpretation. In this paper, the third random field $U=$ $\left(U_{s}\right)_{s \in S}$ is used to incorporate the nonstationary behavior in the posterior distribution, and the pairwise potentials are reconstructed under the combined effect of $X$ and $U$.

Let $S$ be the set of pixels, the label field $X=\left(X_{s}\right)_{s \in S}$ is defined over a finite set of classes $\Omega=(1, \ldots, K)$, and the observed field $Y=\left(Y_{s}\right)_{s \in S}$ represents the observed data of the site $S$. The observed field takes its value from the wavelet coefficients in this paper. In the TMF model, the third field $U=\left(U_{s}\right)_{s \in S}$ is introduced to describe the nonstationary property of the distribution $p(x, y) . U_{s}$ is defined over $\Lambda=\left\{\xi_{1}, \ldots, \xi_{M}\right\}$, where different values in $\Lambda$ represent the different level of stationary attribute of $(X, Y)$. In this letter, a particular case $M=2$ is considered and the field $U_{s}$ is defined in relatively simple way: the sites with intensity variations in their neighborhood are considered as a potential stationarity and labeled as $a$, while sites with imperceptible intensity variations in their neighborhood are considered to be another stationarity and labeled as $b$. Hence, the filed $U$ contains two possible stationarities for any nonstationary images.

At the same time, let us consider that the Markov distribution of $T=(X, U, Y)$ is obtained by assuming that $(X, U)$ is a MRF and its energy is defined as follows:

$$
\begin{aligned}
W(x, u)=\sum_{(s, t) \in C_{H}} \alpha_{H}^{1}\left(1-2 \delta\left(x_{s}, x_{t}\right)\right) \\
-\left(\alpha_{a H}^{2} \delta^{*}\left(u_{s}, u_{t}, a\right)+\alpha_{b H}^{2} \delta^{*}\left(u_{s}, u_{t}, b\right)\right) \\
\quad \times\left(1-\delta\left(x_{s}, x_{t}\right)\right) \\
+\sum_{(s, t) \in C_{V}} \alpha_{V}^{1}\left(1-2 \delta\left(x_{s}, x_{t}\right)\right) \\
\quad-\left(\alpha_{a V}^{2} \delta^{*}\left(u_{s}, u_{t}, a\right)+\alpha_{b V}^{2} \delta^{*}\left(u_{s}, u_{t}, b\right)\right) \\
\quad \times\left(1-\delta\left(x_{s}, x_{t}\right)\right),
\end{aligned}
$$

where $C_{H}$ is a horizontal clique and $C_{V}$ is a vertical clique. Here $\delta\left(x_{s}, x_{t}\right)=1$ when $x_{s}=x_{t}$ and $\delta\left(x_{s}, x_{t}\right)=0$ when $x_{s} \neq x_{t} ; \delta^{*}\left(u_{s}, u_{t}, a\right)=1$ when $u_{s}=u_{t}=a, \delta^{*}\left(u_{s}, u_{t}, a\right)=0$ when $u_{s} \neq u_{t}$; the value of $\delta^{*}\left(u_{s}, u_{t}, b\right)$ has the same form of $\delta^{*}\left(u_{s}, u_{t}, a\right)$. Furthermore, based on the Bayes theorem $p(x, u, y)=p(x, u) p(y \mid x, u)$ and Gibbs energy function $p(X=x, U=u)=\gamma_{z} \exp (-W(x, u))$, the distribution of TMF can be defined as (2):

$$
p(x, u, y)=\gamma_{\mathrm{TMF}} \exp \left[-W(x, u)+\sum_{s \in S} \ln \left(p\left(y_{s} \mid x_{s}, u_{s}\right)\right)\right]
$$

where $\gamma_{\mathrm{TMF}}$ denotes the partition function [15].

\section{Hierarchical TMF Model in Discrete Wavelet Domain}

The observed fields are described with a series of wavelet domain transformations, due to varied characteristics of discrete wavelet transform. Each scale has different characteristic vector for varying resolution, which is more conducive to reflect the nature of the observed field. The wavelet coefficients of $J$ scales discrete wavelet domain of original image and the original image at various spatial resolutions form a multiresolution expression of $J+1$ scales. The acquisition locations of them are $S=\left\{S_{0}, S_{1}, \ldots, S_{J}\right\}$. All the subbands LL, $\mathrm{LH}, \mathrm{HL}$, and $\mathrm{HH}$ obtained by the wavelet decomposition in each scale construct a wavelet coefficient characteristic vector that form a multiscale observation field. The expression of the 
multiscale observed field is $W=\left\{W^{0}, W^{1}, \ldots, W^{J}\right\}$, where $W^{0}$ represents the original image and $W^{n}(1 \leq n \leq J)$ represents the image of the wavelet coefficient vector after $n$ scale wavelet decomposition.

To model the SAR data, Zhang et al. used the generalized Gamma distribution in a hierarchical TMF model [16]. In this paper, the Gaussian MRF (GMRF) [17] model is used for the observed field since the GMRF is more related to radar backscattering sources. In the GMRF, the relationship between the wavelet coefficient vector at each scale and the neighborhood wavelet coefficient vector at the same scale are considered in the construction of spatial interaction parameter matrix.

3.1. Two-Pass Computation of Posterior Distribution. Let $Z=$ $(X, U)$, at each scale $n(0 \leq n \leq J)$, the random variables $\left(Y_{s}^{n}\right)_{s \in S^{n}}$ are conditionally independent of $\left(X_{s}^{n}, U_{s}^{n}\right)_{s \in S^{n}}$, and the distribution is expressed as $p\left(y_{s}^{n} \mid x_{s}^{n}, u_{s}^{n}\right)=p\left(y_{s}^{n} \mid\right.$ $\left.z_{s}^{n}\right)$. The observed field value of the original image $W^{0}$ at site $s$ depends on the segmentation label set $z_{s}^{n}(n=0)$ and the vector of intrascale second-order neighborhood. The conditional probability is as follows:

$$
\begin{aligned}
& f\left(w_{s}^{n} \mid \eta_{s}^{W^{n}}, z_{s}^{n}=k\right)= \frac{1}{(2 \pi)^{B / 2}\left|\Sigma_{k}^{n}\right|^{1 / 2}} \\
& \times \exp \left\{-\frac{1}{2}\left(e_{s}^{n}\right)^{T}\left(\Sigma_{k}^{n}\right)^{-1} e_{s}^{n}\right\}, \\
& n=0,
\end{aligned}
$$

where $B$ is the number of dimensions, $\eta_{s}^{W^{n}}$ is the collection of the wavelet coefficient vectors at the corresponding second-order neighborhood at scale $n$, and $e_{s}^{n}=$ $w_{s}^{n}-\mu_{k}^{n}-\sum_{\tau \in N} \theta_{k, \tau}^{n} \times\left(w_{s+\tau}^{n}-\mu_{k}^{n}\right)$ represents the vector of white Gaussian noise with zero mean at $s$. In addition, $\tau \in N=$ $\{(0,1),(0,-1),(1,0),(1,1),(-1,-1),(-1,1),(1,-1),(-1,0)\}$ represents the position offset between the second-order neighborhood system and the center, as shown in Figure 1. $\mu_{k}^{n}, \Sigma_{k}^{n}, \theta_{k, \tau}^{n}$ are the mean vector, the noise covariance matrix, and the spatial interaction parameter matrix. The wavelet coefficient vectors at the same scale are described as $\theta_{k, \tau}^{n}$, where each $\theta_{k, \tau}$ is a $4 \times 4$ matrix, and it expresses the relationship of the four dimensional wavelet coefficient vectors at the offset value of $\tau$ with reference to the current position. Therefore, the parameters of the observed field can be expressed as $\lambda=\left(\mu_{k}^{n}, \Sigma_{k}^{n}, \theta_{k, \tau}^{n}\right)$.

Hence, the bottom-up pass is performed as follows: firstly, compute the bottom level scale at $n=0$ according to (4):

$$
\begin{aligned}
f\left(y_{s}^{0} \mid z_{s}^{0}\right)= & f\left(w_{s}^{0} \mid \eta_{s}^{W^{0}}, z_{s}^{0}=k\right) \frac{1}{(2 \pi)^{B / 2}\left|\Sigma_{k}^{0}\right|^{1 / 2}} \\
& \times \exp \left\{-\frac{1}{2}\left(e_{s}^{0}\right)^{T}\left(\Sigma_{k}^{0}\right)^{-1} e_{s}^{0}\right\} .
\end{aligned}
$$

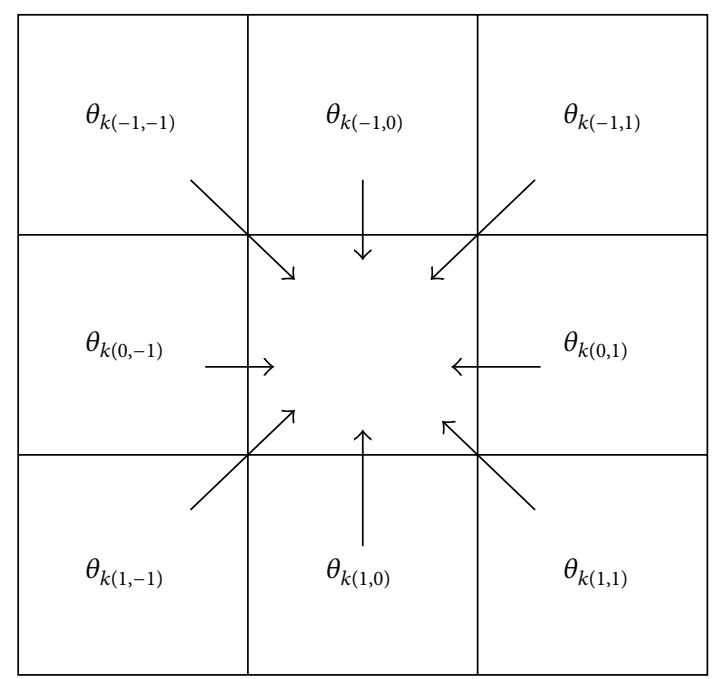

FIGURE 1: Interaction parameters of GMRF model.

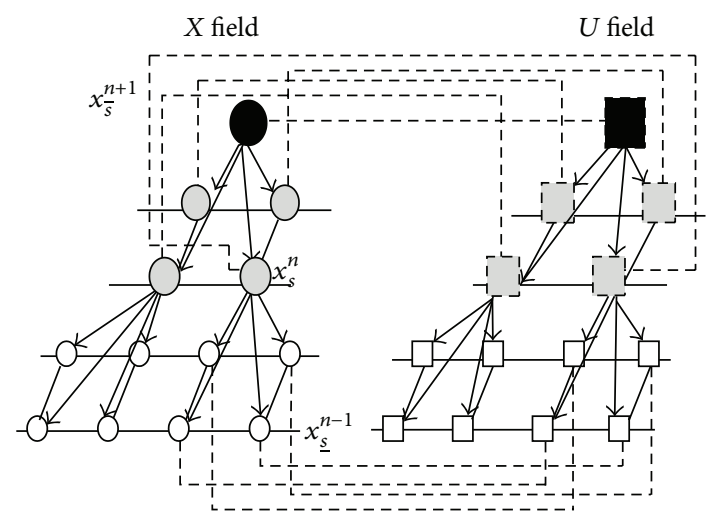

FIGURE 2: Quadtree structure.

Then, for $0<n \leq J$, use the relationship between the current scale and the child scale, as given in (5):

$$
\begin{aligned}
f\left(y_{s}^{n} \mid z_{s}^{n}\right)= & \prod_{s \in S^{n}} f\left(w_{s}^{n} \mid \eta_{s}^{W^{n}}, z_{s}^{n}\right) \\
\times & {\left[\prod _ { r \in \mathcal { c } ( s ) } \left(\sum_{k \in 2 \times \Omega} f\left(y_{\underline{s}}^{n-1} \mid z_{\underline{s}}^{n-1}=k\right)\right.\right.} \\
& \left.\left.\times p\left(z_{\underline{s}}^{n-1}=k \mid z_{s}^{n}\right)\right)\right],
\end{aligned}
$$

where $z_{s}^{n-1}=\left(x_{s}^{n-1}, u_{s}^{n-1}\right)$ and $z_{s}^{n}=\left(x_{s}^{n}, u_{s}^{n}\right)$ are connected in the Markovian chain across the scale in the quadtree structure; see Figure 2. $p\left(z_{s}^{n-1}=k \mid z_{s}^{n}\right)$ is the representation of a first-order Markov chain in interscale of label field, $p\left(z_{\underline{s}}^{n-1}=k \mid z_{s}^{n}=m\right)=\beta_{0} \delta_{k, m}+1-\beta_{0} / M, \beta_{0}$ is the interaction parameter on interscale, $\delta_{k, m}$ is an unit function, and $M$ represents the number of categories; it equals $2 \times \Omega$ in this paper. Finally, the $f\left(y_{s}^{J} \mid x_{s}^{J}\right)$ at the coarsest scale is obtained. 
Then the top-down pass is computed. At the coarsest scale $n=J$, (1) is used to obtain the energy function:

$$
\begin{gathered}
E_{s}^{J}\left(x_{s}^{J}, u_{s}^{J}\right)=W_{s}^{J}\left(x_{s}^{J}, u_{s}^{J}\right) \\
=\sum_{(s, t) \in C^{J}} q_{1}^{J}\left[\alpha_{H V}^{1}\left(1-2 \delta\left(x_{s}^{J}, x_{t}^{J}\right)\right)\right. \\
-\left(\alpha_{a H V}^{2} \delta^{*}\left(u_{s}^{J}, u_{t}^{J}, a\right)\right. \\
\left.+\alpha_{b H V}^{2} \delta^{*}\left(u_{s}^{J}, u_{t}^{J}, b\right)\right) \\
\left.\times\left(1-\delta\left(x_{s}^{J}, x_{t}^{J}\right)\right)\right] .
\end{gathered}
$$

At other scales, according to the Bayesian rule, and under the guidance of the larger scale structure segmentation, the multiscale energy functions of causal TMF model are as follows:

$$
\begin{aligned}
E_{s}^{n}\left(x_{s}^{n}, u_{s}^{n} \mid x_{\bar{s}}^{n+1}, u_{\bar{s}}^{n+1}\right)= & W_{s}^{n}\left(x_{s}^{n}, u_{s}^{n}\right) \\
& +H_{s}^{n}\left(x_{s}^{n}, u_{s}^{n} \mid x_{\bar{s}}^{n+1}, u_{\bar{s}}^{n+1}\right),
\end{aligned}
$$

where the former one is the energy of intrascale and the latter one is with respect to interscale:

$$
\begin{aligned}
H_{s}^{n}\left(x_{s}^{n}, u_{s}^{n} \mid x_{\bar{s}}^{n+1}, u_{\bar{s}}^{n+1}\right)=\sum_{s \in S^{n}} q_{2}^{n} & {\left[\beta_{1}\left(1-2 \delta\left(x_{s}^{n}, x_{\bar{s}}^{n+1}\right)\right)\right.} \\
& +\beta_{2}\left(1-2 \delta\left(u_{s}^{n}, u_{\bar{s}}^{n+1}, a\right)\right) \\
+ & \left.\beta_{3}\left(1-2 \delta\left(u_{s}^{n}, u_{\bar{s}}^{n+1}, b\right)\right)\right] .
\end{aligned}
$$

In (6)-(8), where $\alpha_{H V}^{1}=\left\{\alpha_{H}^{1}, \alpha_{V}^{1}\right\}, \alpha_{a H V}^{2}=\left\{\alpha_{a H}^{2}, \alpha_{a V}^{2}\right\}$, and $\alpha_{b H V}^{2}=\left\{\alpha_{b H}^{2}, \alpha_{b V}^{2}\right\}$, thus, the proposed model parameters are $\eta=\left\{\alpha_{H V}^{1}, \alpha_{a H V}^{2}, \alpha_{b H V}^{2}, \beta_{1}, \beta_{2}, \beta_{3}\right\}$, the first three keep consistency with [18], the last three are the interaction parameters between the interscales. $q_{1}^{n}$ and $q_{2}^{n}$ are the weighted coefficient, according to [19], $q_{1}^{n}=2^{n}+2\left(2^{n}-1\right)$ and $q_{2}^{n}=4^{n}$.

3.2. Segmentation Algorithm Based on the Sequential MAP (SMAP). The SMAP estimator is used to segment the multiscale image [20]. It gives different penalties to the segmentation errors at different scales. The segmentation errors at larger scale have significantly higher effect on the final cost function compared to the errors at smaller scales. This is similar to the human visual system; therefore, the SMAP can improve the performance of the segmentation process. To determine the model parameters, and the segmentation results in the top-down pass, the recursive estimation procedure is expressed as (9)-(12):

$$
\begin{aligned}
& p\left(x_{s}^{J}, u_{s}^{J} \mid y_{s}^{J}\right) \\
& \propto \gamma^{J} \exp \left[-E_{s}^{J}\left(x_{s}^{J}, u_{s}^{J}\right)\right. \\
&\left.+\sum_{s \in S^{J}} \ln \left(f\left(y_{s}^{J} x_{s}^{J}, u_{s}^{J}\right)\right)\right],
\end{aligned}
$$

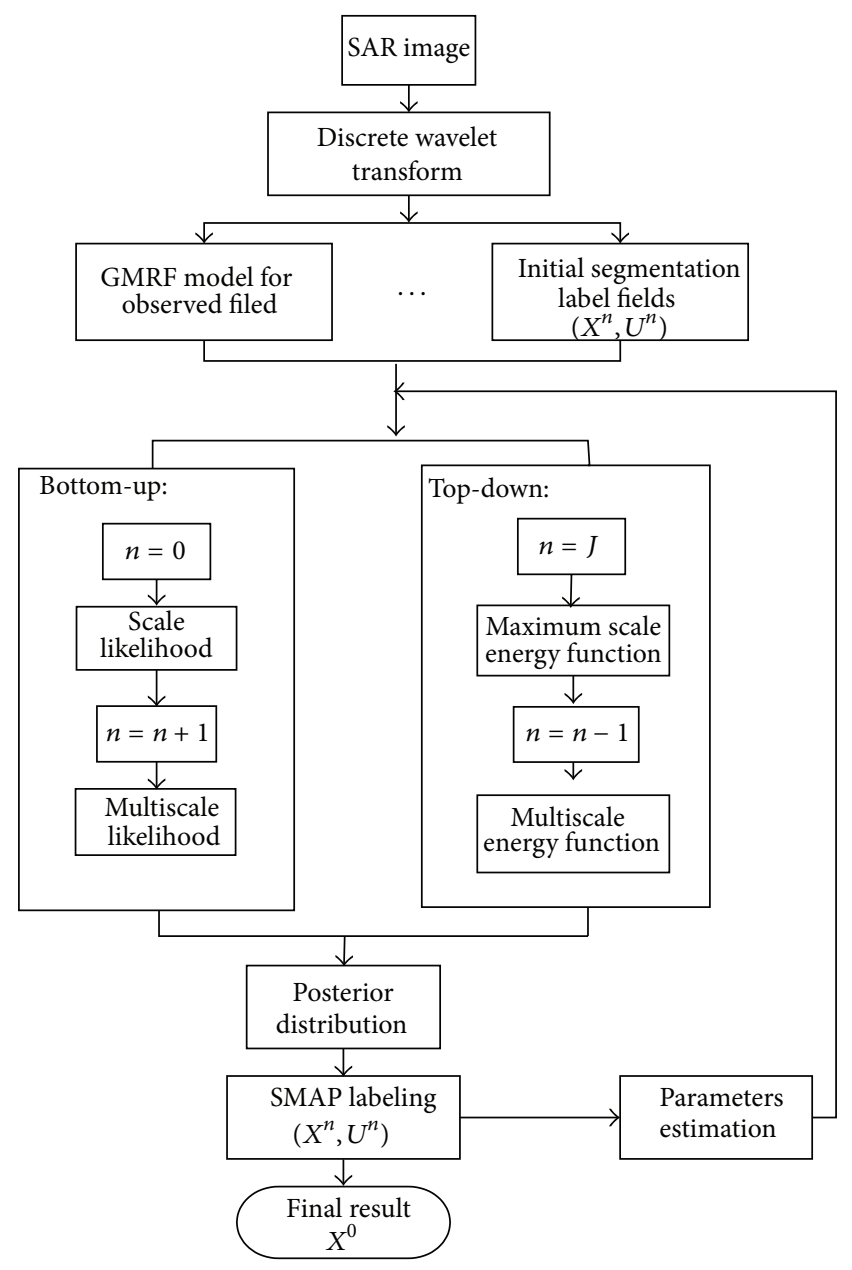

FIGURE 3: Flow diagram of the proposed segmentation method.

$$
\begin{gathered}
\widehat{x}_{s}^{J}=\arg \max _{X_{s}^{J}} \sum_{U_{s}^{J} \in \Lambda} p\left(x_{s}^{J}, u_{s}^{J} \mid y_{s}^{J}\right), \\
p\left(x_{s}^{n}, u_{s}^{n} \mid x_{\partial(\bar{s})}^{n+1}, u_{\partial(\bar{s})}^{n+1}, y_{s}^{n}\right) \\
\propto \gamma^{n} \exp \left[-E_{s}^{n}\left(x_{s}^{n}, u_{s}^{n} \mid x_{\partial(\bar{s})}^{n+1}, u_{\partial(\bar{s})}^{n+1}\right)\right. \\
\left.+\sum_{s \in S^{n}} \ln \left(f\left(y_{s}^{n} x_{s}^{n}, u_{s}^{n}\right)\right)\right], \\
\widehat{x}_{s}^{n}=\arg \max _{X_{s}^{n}} \sum_{U_{s}^{n} \in \Lambda} p\left(x_{s}^{n}, u_{s}^{n} \mid x_{\partial(\bar{s})}^{n+1}, u_{\partial(\bar{s})}^{n+1}, y_{s}^{n}\right), \\
0 \leq n<J .
\end{gathered}
$$

3.3. Parameter Estimation. In the unsupervised segmentation applications, the parameter estimation cannot be performed on the training data. Therefore, the expectationmaximization (EM) algorithm is used for estimation in this paper. In the iterative process of EM algorithm, the efficient maximizing pseudolikelihood (MPL) is selected with the GMRF model parameters. To compute the interaction 


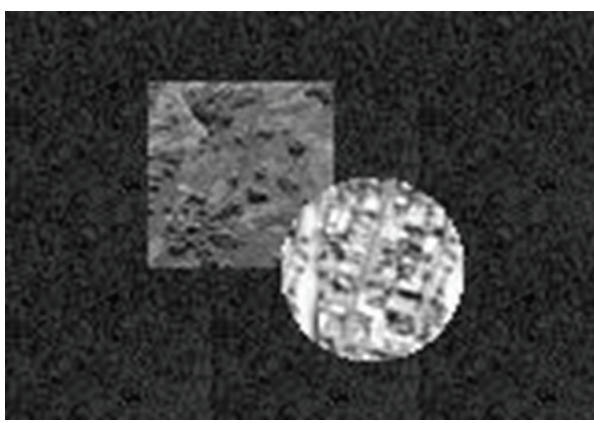

(a) A synthetic SAR image

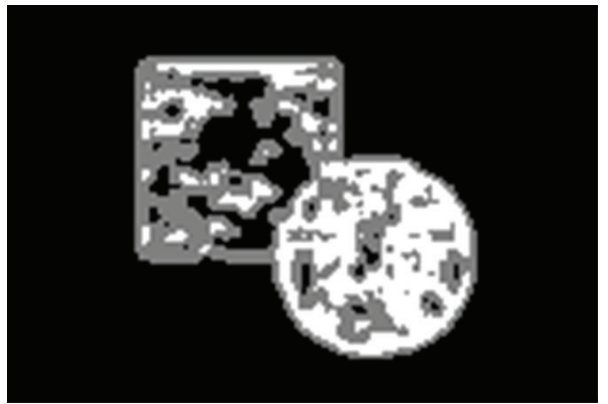

(c) $\mathrm{KMCF}$

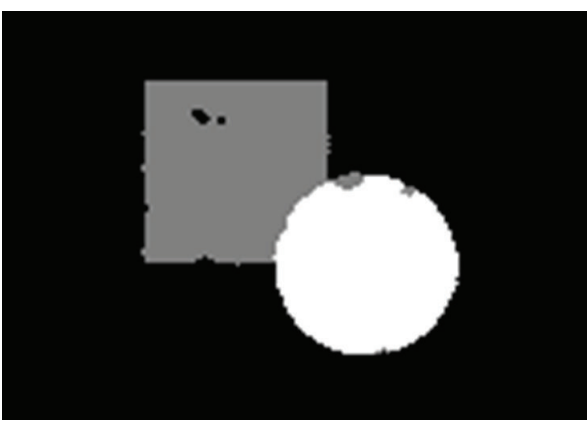

(b) WHTMF

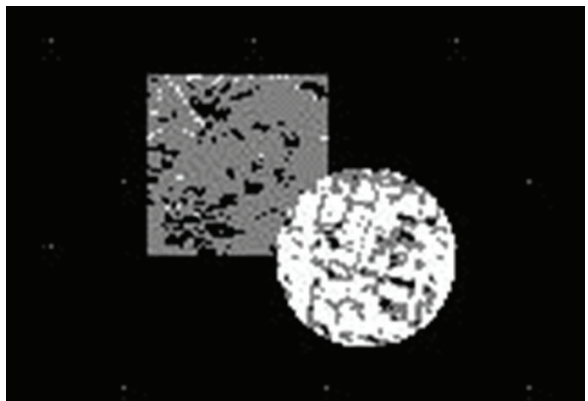

(d) HMF

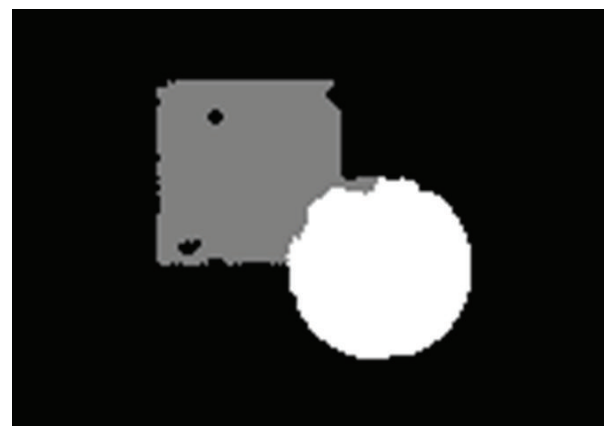

(e) TMF

FIGURE 4: A synthetic SAR image and the segmentation results of different models.

parameters $\beta=\left\{\beta_{0}, \beta_{1}, \beta_{2}, \beta_{3}\right\}$, the EM algorithm is used in similar way as [21]. Finally, in order to obtain the segmentation results, the fast iteration condition mode (ICM) [22] is used with the estimated parameters to maximize (10) and (12). The entire process is shown in Figure 3.

\section{Experiments and Discussions}

4.1. Segmentation Quality Assessment Criterion. In order to evaluate the segmentation quality of the proposed algorithm, the aspects of visual effects and the quantitative indications are considered. From the visual point of view, the algorithm that maintains the local features and preserves the edge information is considered as a better segmentation method. On the other hand, the Kappa coefficient and the classification error rate are mostly used in the quantitative evaluation index [23].

Under the hypothesis that the ideal segmentation result is known, the results of the proposed method are compared with the ideal. The pixels of the class $i$ are classified as class $j$, given as $N_{i j} ; N$ is the total number of image pixels, $N_{i+}=$ $\sum_{j=1}^{K} N_{i j}$ is the number of pixels that are classified into the class $i$ in the classification process, and $N_{+j}=\sum_{i=1}^{K} N_{i j}$ is the number pixels in the actual class $j$ in the image. The Kappa coefficient is given as follows:

$$
\kappa=\frac{N \sum_{i=1}^{K} N_{i i}-\sum_{i=1}^{K}\left(N_{i+} N_{+i}\right)}{N^{2}-\sum_{i=1}^{K}\left(N_{i+} N_{+i}\right)} .
$$

The classification error rate is a statistic index with significant probability. It refers to the probability of each random sample classification that is consistent with the actual classification; it is calculated as follows:

$$
\text { error }=1-\frac{\sum_{i=1}^{K} N_{i i}}{N} \text {. }
$$

4.2. Performance Evaluation on Synthetic SAR Images. The WHTMF model is applied to the segmentation of synthetic 


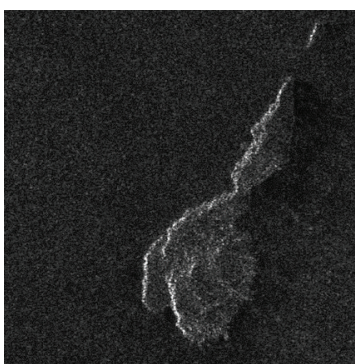

(a)

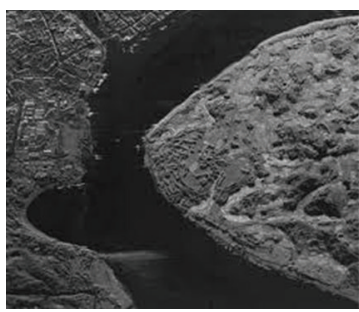

(a)

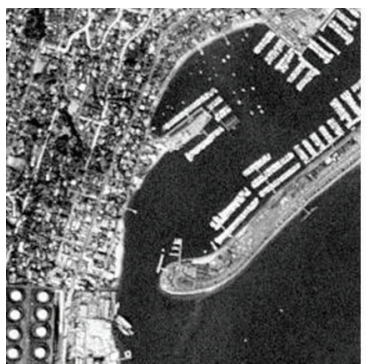

Case 3

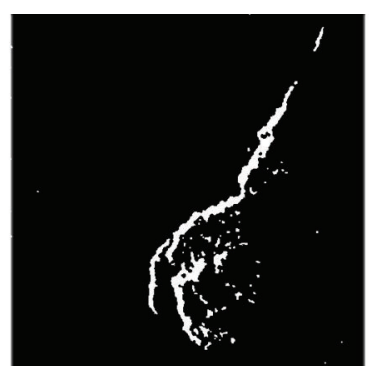

(b)

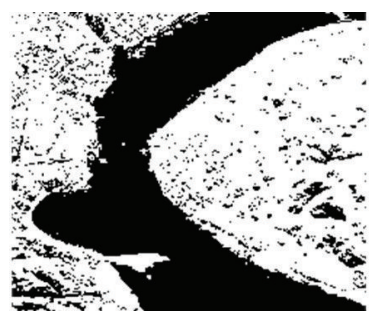

(b)

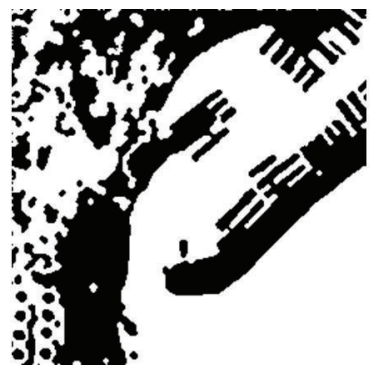

(b)

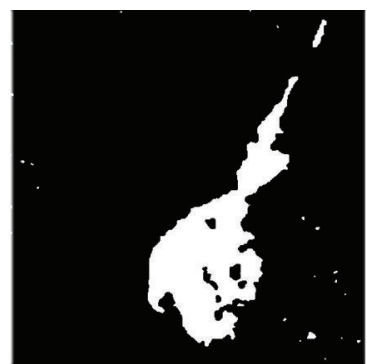

(c)

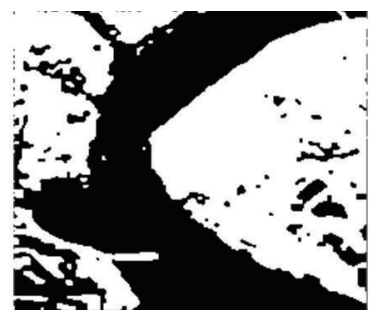

(c)

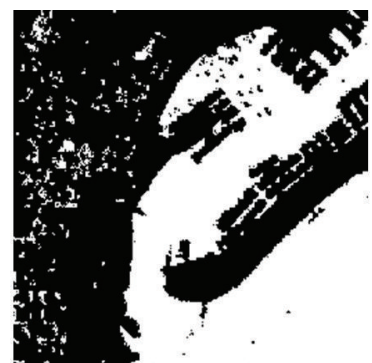

(c)

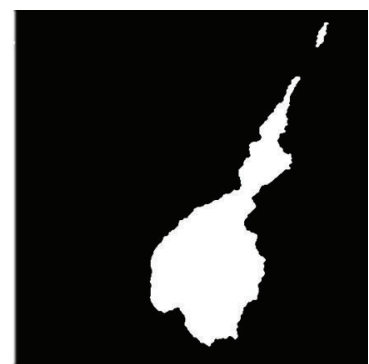

(d)

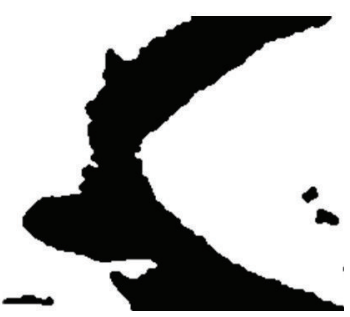

(d)

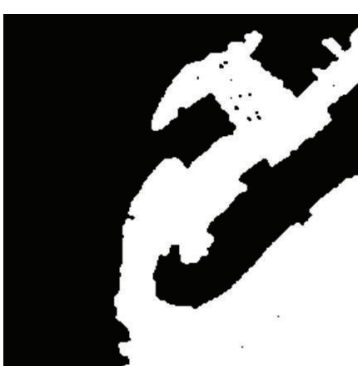

(d)

FIGURE 5: SAR original images and results of segmentation by different models.

SAR image to quantitatively describe the quality of segmentation. The segmentation results are compared with three of the traditional methods as Figure 4: the $K$-means clustering by fusion texture image (KMCF), the classical HMF model, and the TMF model.

It can be seen from Figure 4 that the KMCF method and the classical HMF model segmentation methods are sensitive to noise and the distribution of gray value, while the TMF model and the WHTMF model suppress the noise. It is due to the consideration of the relationship between the pixels in local space. As the multiscale likelihood function and multiscale energy function are considered in WHTMF model, the proposed method performs better than the TMF model. The Kappa coefficient and the classification error rate are calculated and provided in Table 1. The greater of the Kappa coefficient with the lower of classification error rate shows the better segmentation performance. The evaluation results are consistent with the human vision system.

4.3. Experimental Results on Real SAR Images. The segmentation quality of the proposed method and the two efficient selected methods are also evaluated on real SAR images of different scenarios, as shown in Figure 5, where Figure 5(a) shows the original SAR images, Figure 5(b) shows the segmentation results of the HMF model, Figure 5(c)
TABLE 1: Segmentation quality assessment of different methods.

\begin{tabular}{lcc}
\hline Method & \multicolumn{2}{c}{ Criteria of segmentation quality assessment } \\
& Kappa coefficient & Classification error rate \\
\hline KMCF & 0.7483 & 0.0705 \\
HMF & 0.8312 & 0.0647 \\
TMF & 0.9701 & 0.0374 \\
WHTMF & 0.9822 & 0.0081 \\
\hline
\end{tabular}

shows the segmentation results of the TMF model, and Figure 5(d) shows the segmentation results of the WHTMF model.

It can be seen that the segmentation results of the TMF model are better than the MRF model. The TMF model can better suppress the speckle noise and obtains more accurate segmentation compared to the MRF model. This can be attributed to the introduction of the auxiliary field $U$ that incorporates the nonstationary property of SAR images. The MRF model is fairly simple to model the complex nonstationary SAR images. So, the TMF model turns out to be a more accurate statistical model for SAR images in modeling the spatial structures compared to the MRF model.

However, lacking the local structure information as guidance, the TMF model has produced some incorrect 


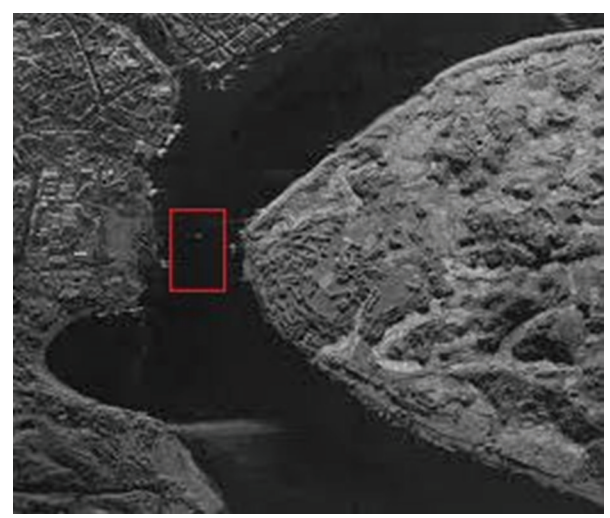

(a) Original SAR image

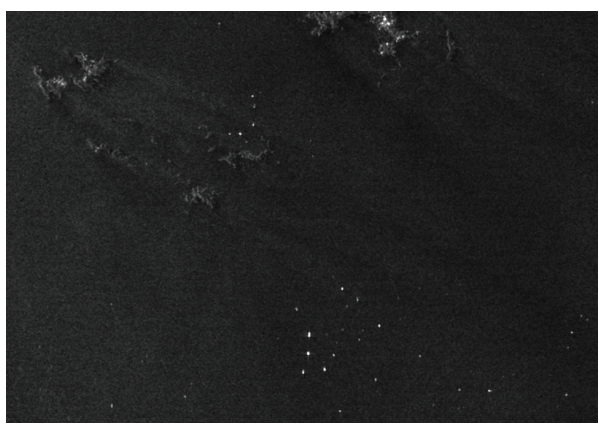

(c) Local magnifying image

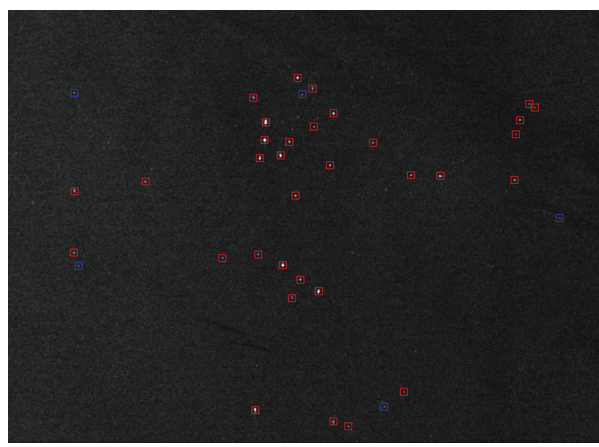

(e) Target recognition

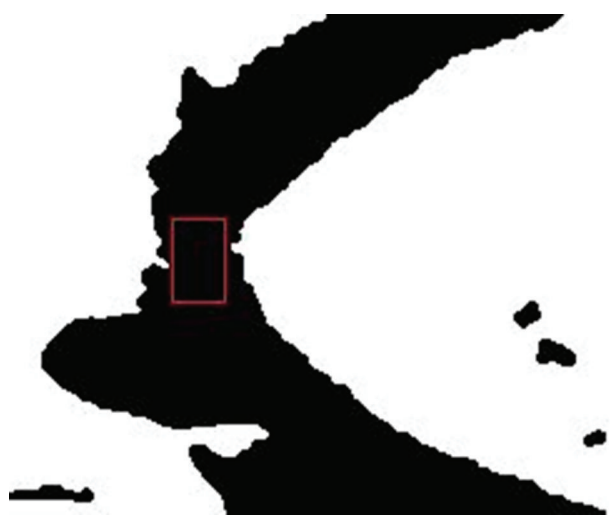

(b) Segmentation of entire scene

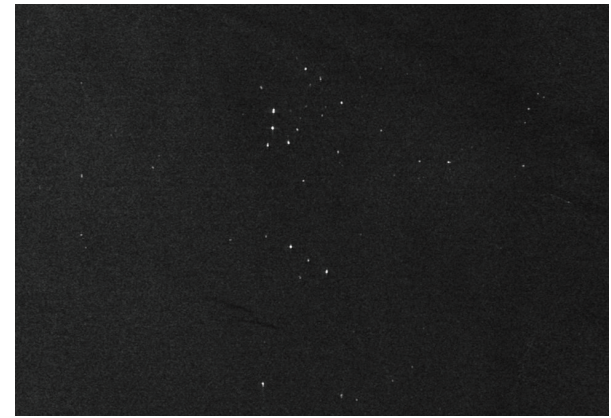

(d) Target extraction

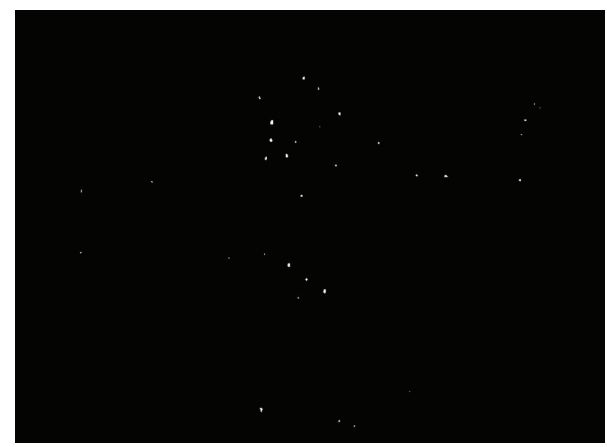

(f) Binary image for information extraction

FIgURE 6: Images in the sea area detection system.

segmentation samples. It is perhaps due to the fact that the TMF model only depends on the limited pixel-level image information. According to the segmentation results, the WHTMF model can achieve better performance than the TMF model. It is observed that it produces significantly less incorrect segmentation samples compared to the TMF. Moreover, the edge information in the result of WHTMF is more accurate. These improvements can be attributed to the interscale dependencies captured by the multiscale energy function and the multiscale likelihood in our algorithm.

The WHTMF model captures the statistical property of the wavelet coefficients, and multiscale likelihoods are computed in the bottom-up pass computations. Combined with the multiscale energy function in the top-down pass computation, the conditional posterior distribution is obtained. The large structure segmentation at coarser scale can serve as a mask and allow the segmentation in finer scale to adjust. Consequently, the segmentation results by the WHTMF model show better performance.

\section{Application on Sea Area Detection System}

Segmentation is the basis of sea area detection; it can provide the coordinates of the sea area from the entire SAR image. The selected region will be sent to the detector that can magnify the region for ship target detection. Recently, the system of sea area detection for ship target has the routine modules as follows. (1) Land isolation module can protect the ships target from the interference of land areas false alarm. (2) Preprocessing module restrains background clutter to highlight the ship target. (3) Target segmentation module separates the ship target from the sea background by 
specific algorithm of ship detection. (4) Target recognition module gets rid of the false target by a priori information of ship target. (5) Information extraction module can apply to the high resolution SAR image to extract the ship target parameters. An example is shown in Figure 6.

Firstly, the land isolation module is used to separate the sea area from the entire scene, shown in Figure 6(b), and this module uses the segmentation method proposed in this paper, which is the significant premise of the system. If the segmentation is not good enough, the false alarm of the land areas will bring the error to the detection threshold, and the latter modules will be influenced by the error; so the result will not be accurate. Secondly, the selected area is preprocessed for despeckle; the despeckle algorithm of speckle reducing anisotropy diffusion (SRAD) [24] is used here. From the local magnifying image as Figure 6(c), it can be see that there are some interferences of sea clutter and scattered islands from the sea background around the ship target. Therefore, use the specific algorithm of ship detection to separate the ship target from the sea background. The selfadaption constant false alarm (CFAR) algorithm is applicable to detect the discrete dynamic ship target. And Figure 6(d) shows the extracted ship target from the background. Then, though a priori information to get rid of the false target, Figure 6(e) labels the false target with blue color. Finally, the binary image of ship targets is obtained to provide parameter information.

\section{Conclusion}

In this letter, a novel unsupervised SAR image segmentation method is proposed that is based on hierarchical TMF in discrete wavelet domain for sea area detection. A Two-pass computation of posterior distribution involves the multiscale likelihood and multiscale energy function, and it effectively captures the global and local image characteristics. The intrascale and the interscale dependences for hierarchical TMF are considered. The experimental results show that the WHTMF model can obtain the optimized parameters and performs better segmentation of the SAR images. We use the WHTMF model in the application of sea area detection system as the first and an important basic step, and the detection of ship target is more accurate and timesaving. However, the WHTMF model studied in this letter is very simple and particularly hypothesizes that the SAR image data are Gaussian model, and this hypothesis can be relaxed to construct more general distribution models in our future research.

\section{Conflict of Interests}

The authors declare that there is no conflict of interests regarding the publication of this paper.

\section{Acknowledgments}

This research is supported by the national natural science fund project of youths science fund: The key technology research of video coding concurrent design and efficient realization (no. 61201238), and the fund project of International Exchange Program of Harbin Engineering University for Innovation oriented Talents Cultivation: The system of SAR anti-jamming effect evaluation (GH20111187).

\section{References}

[1] J. C. Curlander and R. N. McDonough, Synthetic Aperture Radar Systems and Signal Processing, Academic Press, San Diego, Calif, USA, John Wiley \& Sons, New York, NY, USA, 1991.

[2] E. Barthelet, G. Mercier, L. Denise, and S. Reynaud, "Feature based maximum likelihood model inversion for threedimensional building extraction from single high resolution SAR images," in Proceedings of the 32nd IEEE International Geoscience and Remote Sensing Symposium (IGARSS '12), pp. 3600-3603, July 2012.

[3] D. Kong, W. Yang, and Z. Ge, "Active contours driven by local statistical fitting energy for SAR image segmentation," International Journal of Digital Content Technology and Its Applications, vol. 6, no. 14, pp. 469-479, 2012.

[4] O. Lankoande, M. M. Hayat, and B. Santhanam, "Segmentation of SAR images based on markov random field model," in Proceedings of the IEEE International Conference on System, Man and Cybernetics, pp. 2956-2961, October 2005.

[5] G. Dong, N. Wang, C. Hu, and Y. Jiang, "SAR image segmentation combining the PM diffusion model and MRF model," in Proceedings of the 32nd IEEE International Geoscience and Remote Sensing Symposium (IGARSS '12), pp. 4307-4310, July 2012.

[6] L. Lopez, M. Moctezuma, and F. Parmiggiani, "Contextual approach for oil spill detection in SAR images using image fusion and markov random fields," in Proceedings of the 49th Midwest Symposium on Circuits and Systems, vol. 2, pp. 137-139, August 2007.

[7] V. Vidya, H. Choi, and G. R. Baraniuk, "Multiscale SAR image segmentation using wavelet-domain hidden markov tree model," in 7th Algorithms for Synthetic Aperture Radar Imagery, vol. 4053 of Proceedings of SPIE, pp. 110-120, April 2000.

[8] J.-G. Zhang, X.-B. Wen, X. Jiao, and L. Wang, "Multiscale markov random field method for SAR image segmentation," in Proceedings of the 2nd International Congress on Image and Signal Processing (CISP '09), pp. 1-5, Tianjin, China, October 2009.

[9] D. Benboudjema and W. Pieczynski, "Unsupervised statistical segmentation of nonstationary images using triplet Markov fields," IEEE Transactions on Pattern Analysis and Machine Intelligence, vol. 29, no. 8, pp. 1367-1378, 2007.

[10] F. Wang, Y. Wu, Q. Zhang, P. Zhang, M. Li, and Y. Lu, "Unsupervised change detection on SAR images using triplet markov field model," IEEE Geoscience and Remote Sensing Letters, vol. 10, no. 4, pp. 697-701, 2013.

[11] F. Wang, Y. Wu, Q. Zhang, W. Zhao, M. Li, and G. Liao, "Unsupervised SAR image segmentation using higher order neighborhood-based triplet markov fields model," IEEE Transactions on Geoscience and Remote Sensing, vol. 52, no. 8, pp. 5193-5205, 2014.

[12] X. Lian, Y. Wu, W. Zhao, F. Wang, Q. Zhang, and M. Li, "Unsupervised SAR image segmentation based on conditional triplet Markov fields," IEEE Geoscience and Remote Sensing Letters, vol. 11, no. 7, pp. 1185-1189, 2014. 
[13] L. Gan, Y. Wu, M. Liu, P. Zhang, H. Ji, and F. Wang, "Triplet Markov fields with edge location for fast unsupervised multiclass segmentation of synthetic aperture radar images," IET Image Processing, vol. 6, no. 7, pp. 831-838, 2012.

[14] B.-L. Liu, Z. Tian, and M.-T. Ding, "Texture feature-based segmentation of SAR images using a multiresolution pairwise markov-GAR model," Yuhang Xuebao/Journal of Astronautics, vol. 28, no. 3, pp. 677-701, 2007.

[15] D. Benboudjema and W. Pieczynski, "Segmenting non stationary images with triplet Markov fields," in Proceedings of the IEEE International Conference on Image Processing (ICIP '05), pp. 317320, September 2005.

[16] P. Zhang, M. Li, Y. Wu, G. Liu, H. Chen, and L. Jia, "Unsupervised SAR image segmentation using a hierarchical TMF model," IEEE Geoscience and Remote Sensing Letters, vol. 10, no. 5, pp. 971-975, 2013.

[17] S. Krishnamachari and R. Chellappa, "GMRF models and wavelet decomposition for texture segmentation," in Proceedings of the IEEE International Conference on Image Processing, pp. 568-571, October 1995.

[18] P. Zhang, M. Li, Y. Wu, M. Liu, F. Wang, and L. Gan, "SAR image multiclass segmentation using a multiscale TMF model in wavelet domain," IEEE Geoscience and Remote Sensing Letters, vol. 9, no. 6, pp. 1099-1103, 2012.

[19] M. Mignotte, C. Collet, P. Pérez, and P. Bouthemy, "Sonar image segmentation using an unsupervised hierarchical MRF model," IEEE Transactions on Image Processing, vol. 9, no. 7, pp. 12161231, 2000.

[20] G.-Y. Liu, A.-M. Wang, R.-Y. Chen, and Q.-Q. Qin, "Supervised image segmentation method based on tree-structured Markov Random Field in wavelet domain," Journal of Infrared and Millimeter Waves, vol. 30, no. 1, pp. 91-96, 2011.

[21] G.-Y. Liu and A.-M. Wang, "Fuzzy clustering algorithm for integrating multiscale spatial context in image segmentation by hidden Markov random field models," International Journal of Pattern Recognition and Artificial Intelligence, vol. 27, no. 3, Article ID 1355005, 17 pages, 2013.

[22] Y. Wu, M. Li, P. Zhang, H. Zong, P. Xiao, and C. Liu, "Unsupervised multi-class segmentation of SAR images using triplet Markov fields models based on edge penalty," Pattern Recognition Letters, vol. 32, no. 11, pp. 1532-1540, 2011.

[23] C. D'Elia, G. Poggi, and G. Scarpa, "A tree-structured Markov random field model for Bayesian image segmentation," IEEE Transactions on Image Processing, vol. 12, no. 10, pp. 1259-1273, 2003.

[24] Y. Yu and S. T. Acton, "Speckle reducing anisotropic diffusion," IEEE Transactions on Image Processing, vol. 11, no. 11, pp. 12601270, 2002. 


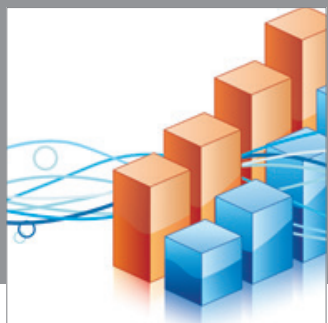

Advances in

Operations Research

mansans

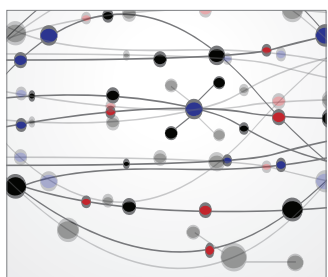

The Scientific World Journal
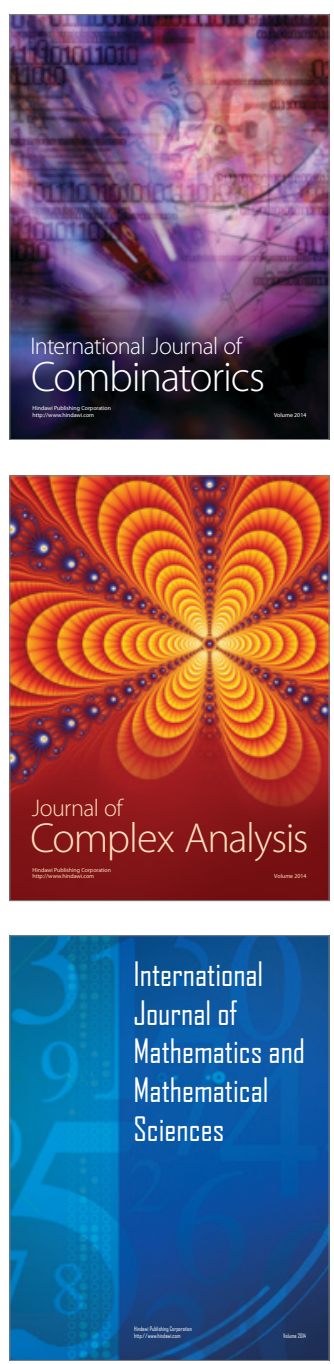
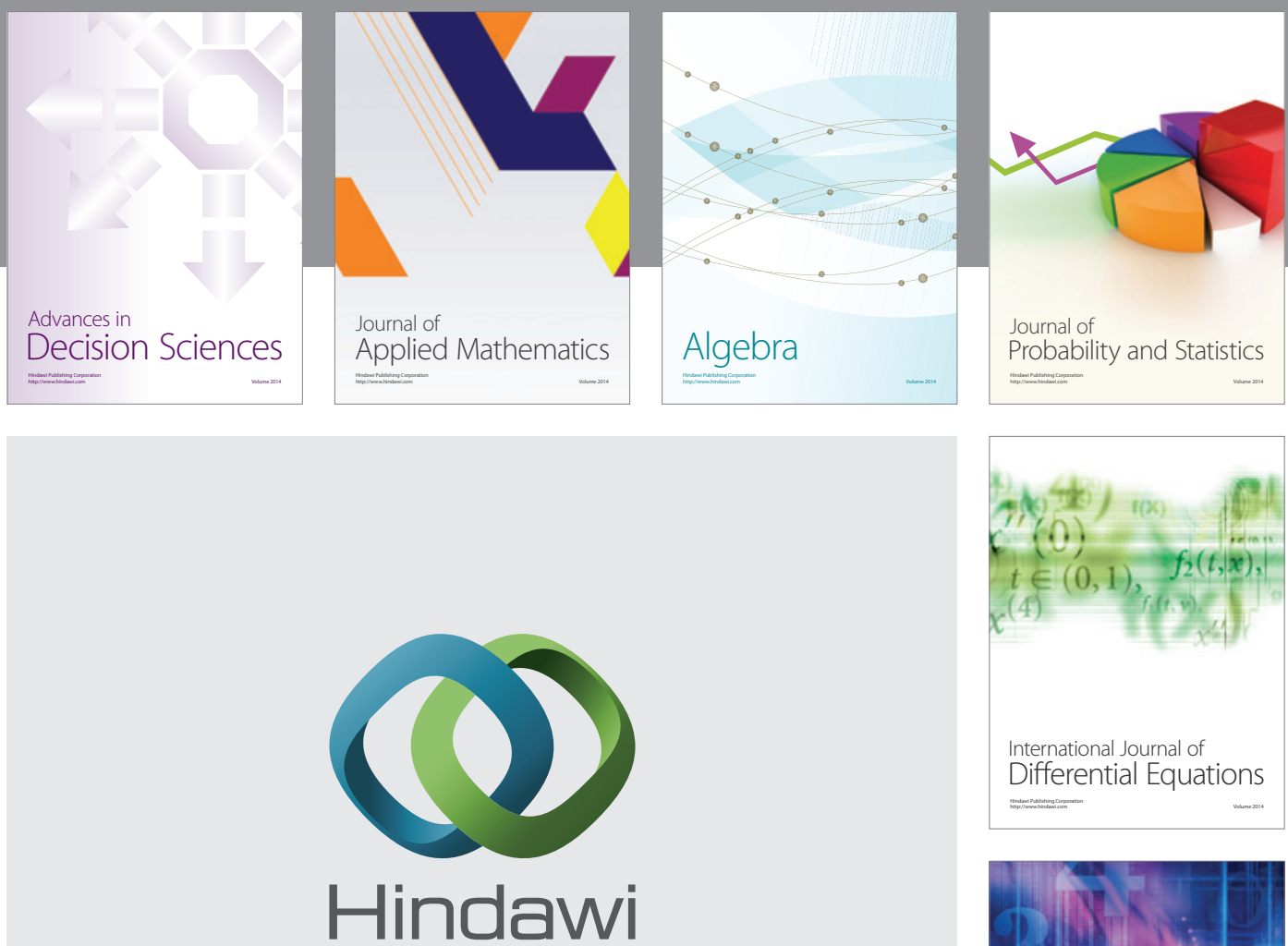

Submit your manuscripts at http://www.hindawi.com
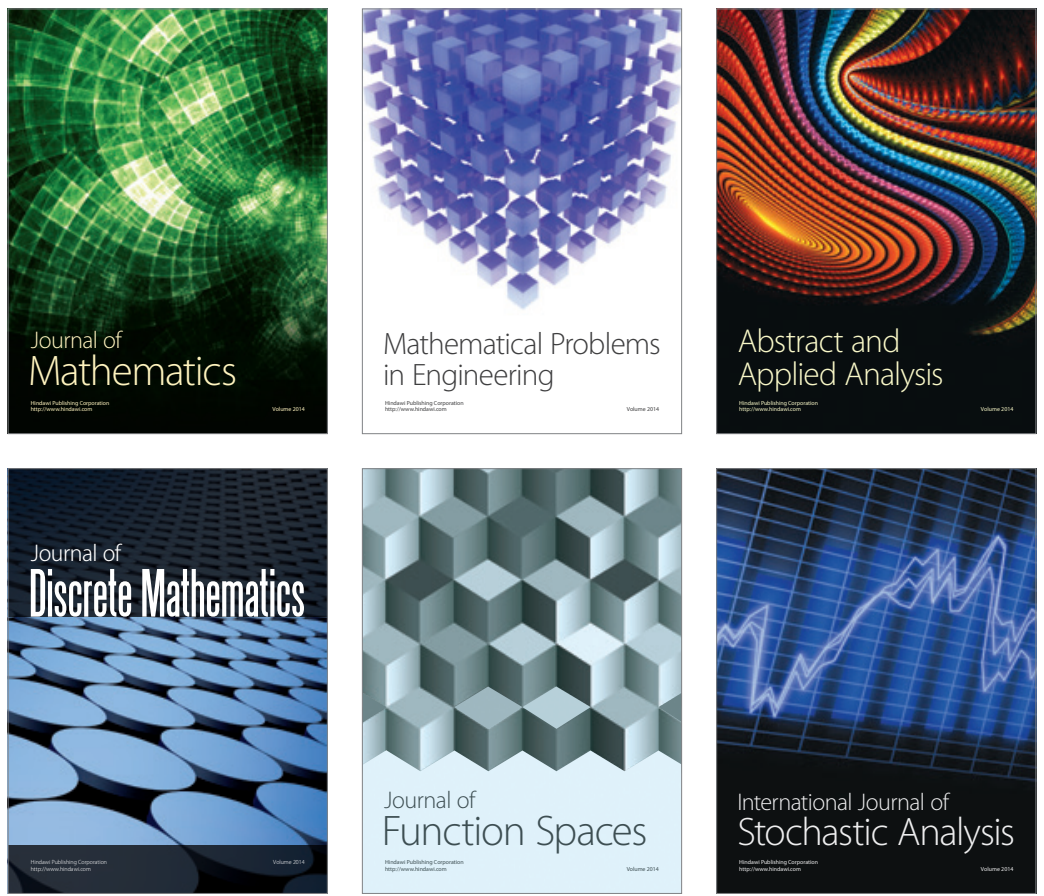

Journal of

Function Spaces

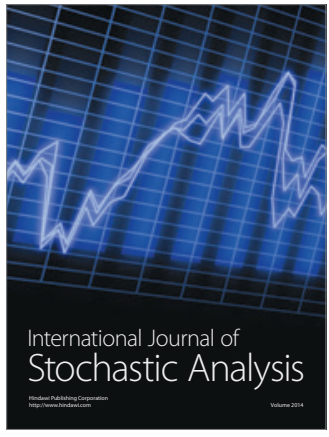

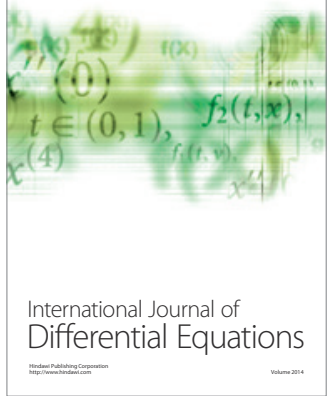
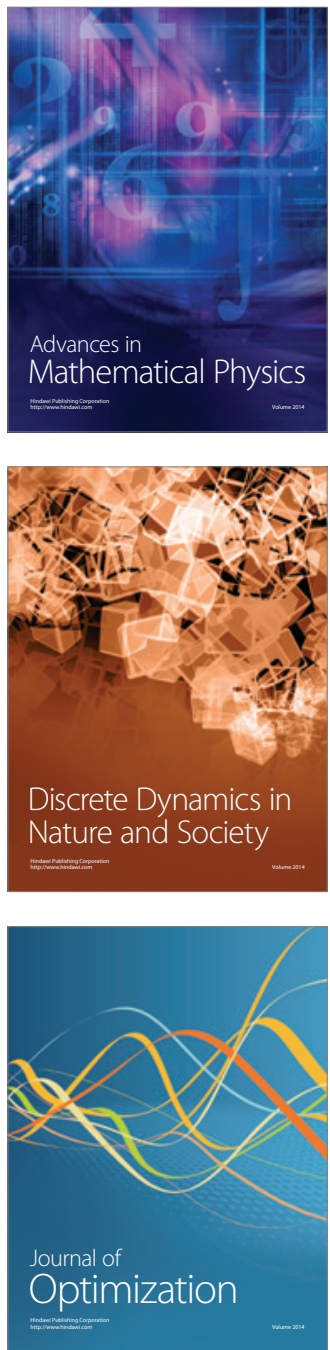